\title{
Abbattista, Lucía (2019). Justicialismo y cultura en la Guerra Fría : EI retorno de Oscar Ivanissevich al Ministerio de Cultura y Educación (Argentina 1974-1975)
}

\author{
María Lucía Abbattista \\ mlabbattista@gmail.com \\ Instituto de Investigaciones en Humanidades y \\ Ciencias Sociales (IdIHCS). Universidad Nacional \\ de La Plata - CONICET / Universidad Nacional de \\ Quilmes, Argentina
}

Cita sugerida: Abbattista, M. L. (2021).

[Revisión de Tesis Justicialismo y cultura en la Guerra Fría : El

retorno de Oscar Ivanissevich al Ministerio de Cultura y

Educación (Argentina 1974-1975)]. Aletheia, 11(22), e097.

https://doi.org/10.24215/18533701e097

Defensa de Tesis: 11 de diciembre de 2019

Directora: María Laura Lenci

Co-Directora: Ana Julia Ramírez

Enmarcada en la historia reciente, inspirada por los estudios sobre el estado desde adentro y con la sensibilidad de los estudios sobre memorias sociales, esta tesis defendida en diciembre de 2019 buscó contribuir con las investigaciones sobre los años del tercer peronismo (1973-1976) a partir del análisis en profundidad de la segunda gestión del cirujano Oscar Ivanissevich en el Ministerio de Cultura y Educación de la República Argentina (1974-1975).

Un punto de partida fue confirmar que aquella gestión, conocida desde entonces con el nombre de "Misión Ivanissevich", ha tenido consecuencias de largo plazo en el campo educativo y cultural argentino y, aunque ha sido extensamente repudiada y denunciada en los ejercicios de memoria sobre la época, no ha sido aún suficientemente investigada, salvo en lo que refiere a algunos terrenos como el universitario.

Por lo general, su llegada imprevista al Ministerio, en agosto de 1974, coincidente con el ascenso de la figura de José López Rega en el gobierno nacional y el despliegue desenfrenado de organizaciones como la Triple A en las calles, se suele explicar como parte de la ofensiva que por entonces se desarrolló contra proyectos, figuras 
y organizaciones de la izquierda del peronismo, como un esfuerzo restaurador de la ortodoxia justicialista, pero su gestión implicó mucho más.

Es cierto que por su historia compartida con Perón, por su silencioso distanciamiento a comienzos de los '50, por ser uno de los pocos sobrevivientes de la primera hora del justicialismo (ajeno a las rencillas que se desataron durante los años de exilio) y por su estilo profesionalista, Ivanissevich podía ser presentado como una encarnación de la ortodoxia doctrinaria, un profeta de "las 20 verdades" del justicialismo, a lo sumo algo anciano para retornar a la acción... Pero Ivanissevich, aún apurado por la convocatoria, concibió su retorno en un marco más amplio. Así como su segunda gestión implicó mucho más que un cambio de rumbo en el área educativa, también fue más que el triunfo de un "ala derecha" en la interna peronista. En un cuarto de siglo el mundo había cambiado y él compartía con intelectuales "paleconservadores" norteamericanos que el orden, las jerarquías y las instituciones de la sociedad estaban en juego en una guerra que era a muerte contra el materialismo marxista.

Por lo tanto, la idea de la tesis es inscribir en un amplio contexto el conjunto de políticas educativas autoritarias y disciplinadoras que desplegó sobre un variado universo de instituciones y actores con la intención de "depurar ideológicamente" el sistema educativo, visibilizando cuáles fueron sus redes, quienes fueron sus principales blancos y precisando también quienes fueron los principales actores que resistieron a sus medidas. De esa forma, la intención es destacar las marcas que dejó una gestión como la de Ivanissevich sobre trayectorias e imaginarios, sobre instituciones y prácticas educativas, artísticas y científicas.

Por su complejidad y dimensión, la investigación sobre esta experiencia se realizó principalmente poniendo en diálogo una variada bibliografía interdisciplinaria con una extensa cantidad de fuentes primarias escritas. Recuperamos expedientes, artículos periodísticos, debates parlamentarios, panfletos y materiales generados durante el desarrollo de algunas de las políticas analizadas. Se trabaja con documentación disponible en papel y en versión digital en el archivo de la Biblioteca del Maestro, en el Centro Nacional de Información y Documentación Educativa y en el Repositorio Institucional del Ministerio de Educación de la Nación; también consultamos el acervo documental sobre el Ministerio de Educación disponible en la Biblioteca Nacional.

Otro Centro de Documentación y Archivo que fue de gran ayuda fue el de la Comisión Provincial por la Memoria de la Provincia de Buenos Aires, que nos aportó legajos producidos por la Dirección de Inteligencia de la Provincia y por otras entidades de la comunidad informativa nacional sobre organizaciones y acontecimientos vinculados con las luchas por el derecho a la educación, así como una compilación sobre copamientos y amenazas a escuelas ocurridas en noviembre de 1974.

Además, fueron consultadas las hemerotecas de la Legislatura de la Provincia de Buenos Aires y la de la Universidad Nacional de La Plata, atendiendo a las secciones sobre política nacional y sobre cultura de distintos diarios y publicaciones periódicas de amplio alcance (como La Nación, La Prensa y La Opinión) que permiten reconstruir tanto la existencia de algunas iniciativas como sus repercusiones públicas inmediatas.

Una tarea similar fue realizada con los números de diferentes publicaciones periódicas que corresponden al período 1973-1976. Con la intención de cubrir las posiciones de distintos sectores hemos consultado ejemplares de Cuestionario, Envido, Latinoamérica, La Causa Peronista, Evita Montonera, El Auténtico, Los Libros, Izquierda Popular, Politica Obrera, El Combatiente, Nuevo Hombre, Las Bases, El Caudillo, Alianza y Cabildo disponibles en sitios como Ruinas Digitales y el Topo Blindado, hallados en archivos personales o editados por la Biblioteca Nacional durante la dirección de Horacio González.

También fueron tomados como fuente los libros de distintos géneros de quienes fueron funcionarios del tercer peronismo, como: El último Perón de Jorge Alberto Taiana, Medio siglo de politica y diplomacia de Benito Llambí, Economía y Política en el Tercer Gobierno de Perón de Carlos Leyba, Perón-Perón de Guido 
di Tella, Peronismo. Militancia y critica (1973-2008) de Nicolás Casullo y El presidente que no fue de Miguel Bonasso.

Y, por supuesto, los escritos del propio Ivanissevich y su familia que fui consiguiendo en librerías de usados. Entre las obras autobiográficas, la más extensa que publicó Ivanissevich se titula Rindo Cuenta. 1893-1973. Dos tomos impresos en 1973 en los Talleres Gráficos del Ministerio de Cultura y Educación, meses antes de que se barajase su retorno. El primer volumen reúne fuentes y relatos sobre su vida como médico cirujano y su etapa de docente universitario. El segundo, incluye fotos, escritos y recortes de la prensa sobre su rol como embajador en Estados Unidos y como Ministro de Cultura y Educación a fines de los años cuarenta. El otro trabajo en el que incursiona en este género es previo. Se trata del libro Junto a mis enfermos, publicado en 1945 por Amorrortu, que consta de 327 páginas. Allí Ivanissevich realiza una suerte de balance de los primeros cincuenta años de su vida, donde homenajea a sus referentes médicos, rememora sus viajes formativos e incluye muchos de los trabajos realizados en el marco de su cátedra y su instituto, que conforman lo que él define como su doctrina médico-quirúrgica. Al igual que en el caso de Rindo Cuenta..., se publicó inmediatamente antes de su paso a la función pública.

Por último, contamos con dos de los libros escritos por su hermana, Magdalena (firma como Magda) Ivanissevich de D'Angelo Rodríguez. El primero, editado por la Librería Huemul, lleva por título La ciudad de mi infancia (1970). El segundo, publicado en 1996, se tituló Memorias de una familia. Los Ivanissevich en Argentina. 1890-1996. En este caso, con 97 años e impulsada por uno de sus sobrino-nietos, la autora se dispuso a narrar con dos objetivos. En primer lugar, responder al interés de los más de trescientos descendientes directos de sus padres que querían "conocer el origen y la formación de ésta, nuestra familia de difícil apellido eslavo y de tan cálidos y auténticos sentimientos argentinos", y, en segundo lugar, para "honrar la memoria" de sus padres y hermanos, "especialmente del único que actuó en política y por eso mismo fue malentendido y hasta denostado" en referencia tácita a Oscar, aunque en verdad no haya sido el único de ellos que actuó en política.

$* * *$

Para cumplir con sus objetivos, la tesis está organizada en cinco capítulos. El Capítulo I "Ivanissevich, entre Sarmiento y Perón” se centra en la biografía de Ivanissevich desde 1893 a 1950, período que incluye su formación como médico cirujano, sus acercamientos a la política y su participación en el primer peronismo con distintos roles: rector-interventor, embajador, secretario y ministro, pero también organizador de actos y productor de emblemáticos símbolos del movimiento. El recorrido atiende a la trayectoria familiar, profesional y política del principal actor de esta tesis, con especial interés por sus posiciones sobre cuestiones claves del área educativa. Aunque la biografía o trayectoria de una figura política no basta para explicar su acción de gobierno, entendemos que permite reunir piezas dispersas del rompecabezas y exponer algunas redes que serán claves en la etapa que es centro de la tesis.

Con el Capítulo II, titulado "El tercer peronismo y la gestión Taiana", se propone un repaso por las principales transformaciones políticas, sociales y culturales ocurridas entre 1955 y 1973, que funcionaron como condición de posibilidad para el retorno del peronismo al gobierno y, concretamente, para el nombramiento de Jorge Alberto Taiana, predecesor de Ivanissevich, en el Ministerio. Allí se repasan cuáles fueron los principales elementos que distinguieron a la política educativa encabezada por Taiana de las experiencias realizadas en el área durante los primeros gobiernos peronistas y los principales focos de conflicto que enfrentó, para dar cuenta de nuevos actores movilizados (diferentes a aquellos de fines de los '40) y del estado en el que diversos ámbitos se encontraban a mediados de 1974.

De esta manera, se llega al Capítulo III: "El retorno de Ivanissevich", corazón de la tesis. Antes de ahondar sobre la segunda gestión del ministro, se analizan algunas producciones de Ivanissevich de fines de los años '60 que ayudan a definir su perfil paleoconservador. Luego, con el afán de construir una mirada panorámica sobre 
el conjunto de "la Misión" se describen en profundidad el escenario en que retorna Ivanissevich al Ministerio, quiénes llegan con él, cuáles son sus principios, las manifestaciones de oposición y las redes de apoyo político y gremial que se articulan en los once meses que se encuentra al frente del área para llevar adelante la cruzada. También se repasan allí las circunstancias que rodearon su renuncia el 11 de agosto de 1975, celebrada por numerosos actores como un triunfo de la movilización popular.

Sobre esa base, los últimos dos capítulos se tratan de abordajes en profundidad de los principales territorios donde se desplegó su administración, con la intención de identificar sus diferentes modalidades y ritmos de intervención y disciplinamiento, los grupos concretos con que artículo en cada ámbito y también, especialmente, los nombres e historias de algunas de sus numerosas víctimas.

En el primer caso "El disciplinamiento de las universidades" (Capítulo IV), se trabaja el ámbito universitario y el sistema científico construido en torno a las universidades públicas en los años previos. Sobre las instituciones de educación superior hay buenas investigaciones previas, aunque priman las perspectivas locales y regionales. En este caso, se intenta articular la bibliografía disponible sobre el impacto de "la Misión” en diferentes universidades del país y el rol del movimiento estudiantil en la resistencia, con trabajos contemporáneos de denuncia y algunos hechos reconstruidos a partir de la prensa, que han sido menos atendidos, como la desarticulación de la Secretaría de Ciencia y Técnica erigida durante la gestión Taiana y el anteproyecto de ley universitaria elevado por la Confederación General Universitaria al ministro en julio de 1975.

Por último, en el Capítulo V: "El antagonismo con CTERA", se reconstruye la dinámica menos conocida de su intervención en el terreno de la lucha gremial de los y las docentes, con un extenso repertorio de medidas que incluyen el reconocimiento a la Unión de Docentes Argentinos y el intento por aniquilar la experiencia de la Confederación de Trabajadores de la Educación de la República Argentina. Allí se confrontan, a su vez, dos concepciones sobre el magisterio y sobre los derechos laborales de los trabajadores de la educación.

El análisis de la intervención disciplinadora sobre las Universidades y de los modos que adoptó la confrontación con los trabajadores de la educación confederados en CTERA permiten visualizar cómo el ideario de un peronista clásico se entrecruzó hacia mediados de los setenta con la impronta represiva que avanzaba en el Cono Sur, transformando el retorno de Ivanissevich en un cataclismo político y cultural. Algo similar permitiría observar su despliegue contra el movimiento estudiantil secundario, que excede las posibilidades de esta tesis.

$* * *$

A pesar de la exhaustividad procurada, la tesis aborda una etapa sobre la que constantemente se encuentran nuevas fuentes y se recuperan nuevos testimonios que permiten revisar las interpretaciones previas. En cualquier caso, con el carácter provisorio que puede tener cualquier conclusión, hay cinco ideas suficientemente acreditadas sobre la singularidad de esta gestión que se construyen en los diferentes capítulos y se pasan en limpio en las conclusiones:

En primer lugar, que entre su primera gestión (1948-1950) y la que nos ocupa no hubo grandes transformaciones en el marco ideológico o doctrinario del ministro. Más bien se puede observar la incorporación de diferentes elementos del nuevo contexto para reforzar las posiciones del pasado, o para revestir de mayor gravedad o seriedad a la propia acción. Esto pudo verse no sólo en la persistencia de ideas generales como la reivindicación de aquella ortodoxia justicialista que había contribuido a moldear a fines de los años '40, sino también en dimensiones más específicas como la oposición a la participación estudiantil en las instituciones educativas, por ejemplo, que se refuerza ante la cultura juvenil contestataria de los años '60 y '70. Articulando sus viejas concepciones con nuevas lecturas, la "misión Ivanissevich" se propuso -a partir del Mensaje brindando en el emblemático Teatro Colón- el disciplinamiento social y la refundación 
cultural del país y, en poco menos de un año, llevó adelante diversas iniciativas que articularon la pluma, la cruz y la espada.

En segundo lugar, que los crímenes parapoliciales, los atentados, las intimidaciones, la prisión política y la condena al exilio de numerosas figuras del sistema educativo y científico, fueron claves para el avance de las políticas de esta gestión y para asestar constantes golpes a las voces y los espacios colectivos de resistencia. Aunque nos falten pruebas para señalar su coordinación desde las oficinas del ministerio, hay indicios que permiten suponer la aprobación de estas acciones, porque están claras las líneas de contacto entre las autoridades educativas y organizaciones como la CNU, dispuestas a llevar adelante, por cualquier medio, la cruzada contra "los infiltrados marxistas" en el peronismo y otras expresiones de izquierda. Por eso, aunque en esta tesis solo me concentro en dos grandes áreas para analizar los efectos del retorno de Ivanissevich (el ámbito universitario -en términos amplios- y el activismo gremial docente), en la medida en que pude ir identificando algunas modalidades represivas que fueron concurrentes y corresponsables con "la Misión" por las transformaciones que se producen, se intenta recuperar en la exposición los nombres propios de quienes fueron sus víctimas y el ritmo que algunas detenciones, algunos secuestros y algunos crímenes particulares imprimieron a la dinámica política de la etapa.

En tercer lugar, que el antagonismo entre las políticas culturales desplegadas por la gestión de Ivanissevich y las promovidas por el ministerio de Jorge Taiana fue muy marcado, especialmente en lo que hace a la pluralidad ideológica con que Taiana armó su equipo de funcionarios -fiel reflejo del clima frentista del peronismo de 1973 y de sus redes-, la concepción sobre la participación juvenil y el protagonismo popular en las instituciones educativas, en el imaginario histórico y, muy especialmente, en la idea de Ivanissevich sobre la verticalidad y las jerarquías.

En cuarto lugar, que Carlos Frattini, su segundo en el Ministerio, fue una figura clave en ambas gestiones y que sus redes fueron el principal vehículo del retorno de Ivanissevich al Ministerio, aunque las fuentes sobre él sean considerablemente menos abundantes -por no decir directamente escasas- y, por lo tanto, su rol protagónico en la gestión haya quedado opacado o desvanecido con el paso del tiempo. De hecho, están más presentes en el imaginario público y en las investigaciones de historia reciente figuras como Alberto Ottalagano o Remus Tetu, rectores interventores de universidades nacionales, que él. En este sentido, queda apuntada en la tesis la necesidad de muchas y nuevas investigaciones para comprender el lugar de Frattini dentro del peronismo porteño, su papel en la gestión municipal de José Embrioni y sus redes con organizaciones sindicales y juveniles de la derecha peronista, que permitan remarcar el papel que jugó en la constitución del entramado de la represión ilegal sobre el ámbito educativo.

En quinto lugar, que frente a todas las medidas puestas en marcha por la dupla de la cartera educativa, fue enorme el repertorio de acciones de resistencia. En esta tesis pude identificar y señalar algunas grandes oleadas de cuestionamientos, que muestran que el ejercicio de la oposición de amplios sectores fue constante, aunque al comienzo fragmentario y en apariencia no alcanzaba para hacer mella sobre el bloque gobernante. Sin embargo, tras las jornadas de lucha de junio de 1975 y la llamada "crisis de julio" del gabinete nacional, la articulación de núcleos del amplio frente opositor con algunos sectores de la CGT, construyeron mejores condiciones para la expresión de las voces de impugnación. Asimismo, la acción unitaria -tras arduas negociaciones- de diferentes fuerzas políticas y la constitución de organizaciones como las coordinadoras de estudiantes secundarios (a emulación de las coordinadoras interfabriles), con demandas contra la carestía de la vida, del transporte, de las condiciones para el estudio, permitieron teñir el clima en que se produjo su renuncia de la idea de triunfo y formular esperanzas de cambio de época entre quienes habían sido los principales blancos de su administración. De hecho, desde esa perspectiva la tesis busca sumar un elemento de comprensión para entender la virulencia con que las fuerzas represivas de la última dictadura actuaron contra delegados estudiantiles secundarios y dirigentes de la CTERA, por ejemplo, porque a pesar de todas las medidas que desplegaron durante el gobierno de "Isabel" Perón, no sólo no habían logrado someterlos, 
sino que habían llegado a fines de 1975 con mayores niveles de organización y efervescencia a partir de la resistencia a la "Misión Ivanissevich". 